\title{
Francesco VERDE, Elachista. La dottrina dei minimi nell'epicureismo
}

Alain Gigandet

\section{OpenEdition}

Journals

Édition électronique

URL : https://journals.openedition.org/philosant/855

DOI : 10.4000/philosant.855

ISSN : 2648-2789

\section{Éditeur}

Éditions Vrin

\section{Édition imprimée}

Date de publication : 1 novembre 2014

Pagination : $349-352$

ISBN : 978-2-7574-0855-

ISSN : $1634-4561$

Référence électronique

Alain Gigandet, «Francesco verde, Elachista. La dottrina dei minimi nell'epicureismo », Philosophie antique [En ligne], 14 | 2014, mis en ligne le 01 novembre 2018, consulté le 03 décembre 2022. URL : http:// journals.openedition.org/philosant/855; DOI : https://doi.org/10.4000/philosant.855

\section{(2) $\odot \Theta \Theta$}

Creative Commons - Attribution - Pas d'Utilisation Commerciale - Pas de Modification 4.0 International - CC BY-NC-ND 4.0

https://creativecommons.org/licenses/by-nc-nd/4.0/ 
sens de «premier », tandis que le deuxième (Alex., in Metaph., p. 266, 2-14) établit de façon orthodoxe qu'il y en a seulement une et un seul sens de «premier $\gg$.

Pour conclure, cet ouvrage fait connaître à un public élargi les actes du séminaire du Centre Léon Robin, tout en constituant un instrument précis pour le travail spécialisé sur une série de questions cruciales pour la pensée aristotélicienne. La discussion en profitera sans doute beaucoup.

Pinelopi SKarsouli

CNRS, UPR 76, Centre Jean Pépin

Francesco VERDE, Elachista. La dottrina dei minimi nell'epicureismo, Louvain, Leuven University Press (De Wulf-Mansion Centre Series I), 2013, XIV + 440 pages, ISBN 9789058679475.

À un regard superficiel et peu averti, cet ouvrage pourrait sembler ressortir au genre du « presque tout sur presque rien » : un élément extrêmement pointu et technique de la doctrine épicurienne, la théorie des minima, devient l'objet d'une enquête qui, en 354 pages, va en épuiser toutes les dimensions : textuelle, doxographique, conceptuelle, systématique, historique, exégétique, etc. Mais il est entendu, en réalité, que le problème des elachista ne constitue en rien un détail de l'histoire de l'épicurisme, c'est même un apport essentiel de l'ouvrage de Francesco Verde (ci-après FV) que d'établir son importance de manière définitive.

L'idée de minimum semble fondatrice de l'atomisme en un premier sens: l'insécable représente en effet une limite à la réitération de l'opération de division d'un corps sensible. On peut considérer qu'une telle limite définit un minimum corporel, le plus petit élément physique assignable : l'atome (Lucrèce, I, 748 : in rebus minimum consistere). Lorsque Eusèbe de Césarée, citant Denys d'Alexandrie, nomme l'atome d'Épicure elachiston, peut-être, comme le suggère FV, commet-il une confusion entre l'atome lui-même et ses parties (p. 22-23), mais même si c'est le cas, celle-ci peut s'expliquer par une situation en elle-même équivoque. C'est cette complexité qui constitue le sujet même de l'ouvrage de FV. Elle tient à ce que, repoussant d'un cran l'assignation du minimum, Épicure a cru bon de distinguer des parties constitutives de l'atome lui-même. La nature de cette décision, son motif, sa portée, son destin historique sont systématiquement questionnés en trois grandes étapes, qui donnent lieu (I) à une analyse philologique et exégétique des sources premières de la théorie des minima : la Lettre à Hérodote d'Épicure et le De rerum natura de Lucrèce; (II) à une reconstitution du contexte historique de cette doctrine, essentiellement académicien, péripatéticien et mégarique; (III) à une étude de son développement à l'intérieur du Jardin, en relation avec les recherches géométriques dont témoignent plusieurs sources.

I. La lecture de Hrdt. 56-59 et de Lucrèce I, 599-634 et II, 481-499 est avant tout soucieuse d'inscrire ces textes dans une vision, non pas statique, mais dynamique de la doctrine épicurienne, c'est-à-dire de rester sensible aux évolutions possibles d'Épicure lui-même et de ses successeurs. Cette perspective continuera à prévaloir dans la suite de l'ouvrage. 
L'enjeu du passage-clé de $H r d t$., à travers les questions connexes de la grandeur des atomes et de la limitation du nombre de leurs formes, est de répondre aux objections anti-démocritéennes d'Aristote. L'auteur insiste à raison sur l'importance de la méthode d'inférence sémiotique qui permet d'établir l'existence et les caractéristiques des parties de l'atome par un lien d'analogie contrôlée avec le minimum sensible. Il en ressort, en particulier, contre Giussani entre autres, que les minima atomiques sont corporels et non pas simplement intelligibles (voir aussi p. 101103.). Épicure doit établir que les parties minimales constituent matériellement l'atome, tout en se gardant de faire de celui-ci un agrégat.

L'auteur souligne à raison que la démarche de Lucrèce au chant I, pour confirmer la thèse d'Épicure, n'en diffère pas moins de celle du maître. L'existence d'un extremum cacumen du corps invisible semble ici admise sans inférence (j'observe cependant que celle-ci sera établie en I, 748-752), l'intention étant de garantir l'absolue cohérence de l'atome (solida simplicitas), à quoi s'associe son éternité. C'est donc sur la cohésion des parties minimales qu'insiste avant tout le poète. Une difficulté connexe concerne le poids des minimae partes, dont Lucrèce, estime l'auteur, dit qu'elles sont dépourvues : comment alors expliquer le poids de l'atome (p. 93-94) ? J'objecterai pour ma part à cette approche que ... quae nullis sunt partibus aucta, / non possunt ea quae debet genitalis habere / materies, varios connectus, pondera... semble dire précisément le contraire : c'est parce que l'atome est doté de parties qu'il peut, entre autres, être pesant.

DRN, II, 481-499 fait intervenir les minimae partes dans une perspective différente, celle de la variété des figures de l'atome, ce qui nous ramène à $H r d t$. tout en apportant des précisions intéressantes sur la grandeur et la disposition desdites parties.

Au total, l'intérêt des analyses lucrétiennes est de mettre en valeur un enjeu majeur de la théorie des parties minimales, celui de la rigoureuse simplicitas de l'atome.

II. Reconstituer le contexte historique de cette doctrine impose une clarification préalable. La notion épicurienne de minima est essentiellement complexe. Elle se décline en une variété de modalités difficiles à articuler : minimum dans la sensation, dans l'atome, mais aussi minima spatiaux et temporels.

La question des minima temporels est particulièrement épineuse. Plusieurs spécialistes ont nié à raison l'existence de tels minima dans Hrdt. Mais les sources doxographiques amènent à se demander s'il n'y a pas eu évolution ultérieure d'Épicure sur ce point. Quant au problème des minima spatiaux, il a pour toile de fond la question discutée d'une géométrie épicurienne anti-euclidienne.

Cette intrication des problèmes est confirmée par la littérature secondaire dans sa restitution du contexte théorique des constructions épicuriennes : paradoxes du mouvement de Zénon, Timée de Platon, lignes indivisibles de Xénocrate, actualité du mouvement de Diodore Cronos, problématique aristotélicienne du continu. En ressort la grande complexité du rapport d'Épicure à Démocrite. Comme les sources pour ces influences sont très minces, FV se montre ici attentif tant à la terminologie d'Épicure qu'à l'analyse conceptuelle de ses arguments, afin d'identifier les références implicites et de reconstituer des parallèles précis. 
Un réexamen des travaux qui ont fait date à cet égard (notamment ceux de Philippson, Furley, Isnardi Parente, Sedley) vient confirmer la dette critique et même terminologique d'Épicure à l'égard d'Aristote pour ce qui concerne la théorie des limites et des parties indivisibles. Les arguments dialectiques de Diodore Cronos touchant le mouvement influencent ce dialogue contradictoire avec Aristote.

En résumé, la signification théorique de la doctrine des parties minimales réside dans la nécessité éprouvée par Épicure (a) de répliquer aux paradoxes de Zénon sur le mouvement, (b) de se garantir contre la divisibilité tant physique que théorique, (c) de justifier la limitation des formes atomiques, (d) de garantir la cohésion des atomes dans l'agrégat en fondant la possibilité du contact réciproque.

III. Le point de vue «dynamique » adopté par l'auteur le conduit enfin à rechercher dans les écrits des successeurs d'Épicure les signes d'une reprise originale, d'un « développement doctrinal» du problème des elachista. Les (rares) sources mettent la recherche sur la voie d'une interprétation géométrique des minima spatio-temporels: l'auteur retrouve donc le problème historique de l'existence d'une « géométrie épicurienne » alternative à celle des mathématiciens euclidiens.

Cette piste est suivie, après un rappel et une discussion du rejet par Épicure des sciences qui, comme la géométrie, ne contribuent pas à la connaissance de la nature, d'abord par le biais d'une étude des témoignages sur Polyène de Lampsaque. Celui-ci a pu s'efforcer de rendre la géométrie compatible avec les thèses épicuriennes, ou bien s'employer à constituer une « géométrie alternative » proprement épicurienne.

La démarche de Démétrius Lacon, quant à elle, semble avoir consisté à considérer la définition euclidienne d'un être géométrique, par exemple l'angle, pour en entreprendre la réfutation en référence aux principes épicuriens. Mais l’intérêt des épicuriens pour la géométrie se limitait-il à cette dimension négative ? Ils ont dû voir aussi l'intérêt de ses principes pour la science de la nature, notamment la théorie du mouvement, comme cela semble être le cas pour Zénon de Sidon.

Sous réserve des incertitudes liées à la précarité des sources, on assiste très vraisemblablement après Épicure (et peut-être déjà chez ce dernier) à un déplacement de la théorie des minima de la sphère atomique stricte à celle de l'espace et du temps, une réinterprétation de la géométrie étant mise au service de l'élucidation des concepts physiques.

Ce simple résumé ne rend que très imparfaitement justice à l'ampleur et à l'érudition du travail de FV, qui mobilise de manière quasiment exhaustive les ressources de la littérature critique, depuis les travaux classiques jusqu'aux plus contemporains, pour éclairer à la fois l'exégèse des textes, la reconstruction des problèmes, l'arrière-plan historique et les hypothèses concernant les influences, réinterprétations, polémiques, susceptibles d'éclairer l'histoire épicurienne du concept de minima. Le même souci d'exhaustivité caractérise la constitution du corpus des sources originales et doxographiques. Des perspectives précieuses sont ouvertes sur le vocabulaire technique de l'épicurisme replacé dans ses divers contextes (voir, notamment, l'étude des termes onkoi, amere, peras, metabasis, perilepsis, ou encore, en référence à Lucrèce, simplicitas, stipare, conciliare). Enfin, le souci de contextualiser de la manière la plus large problèmes et thèses amène l'auteur à recon- 
struire de larges séquences historico-conceptuelles touchant des questions aussi diverses que les apories du mouvement, les conceptions continuistes et discontinuistes de l'espace et du temps, la problématique physique et géométrique des lignes indivisibles, les rapports entre les idées d'angle et de minimum, etc.

Ce foisonnement, avec la profusion de notes, le caractère digressif de certains développements, quelques redondances, qui en sont autant de conséquences, peut rendre difficile la lecture en continu de l'ouvrage. Celui-ci n'en constitue pas moins pour le chercheur et même simplement pour le lecteur intéressé à l'histoire de la philosophie et de la science de l'Antiquité une ressource exceptionnelle.

Alain GIGANDET

Université Paris Est Créteil-Paris 12

Voula Tsouna, Philodemus, On Property Management, Atlanta, Society of Biblical Literature, 2012 (Writings from the Greco-Roman World, 33), xlv + 125 pages, ISBN 978-1-58983-667-9.

Sur le modèle du On Death $I V$ (De morte) de Philodème, publié chez le même éditeur en 2009 par W. B. Henry, cet ouvrage propose, après une introduction de trente-quatre pages, une traduction - fondée sur l'édition donnée par Christian Jensen en 1906 pour Teubner - de cet écrit de Philodème tenu par lui comme un livre sur l'économie. Même si dans les « Acknowledgements » (p. vii) V. Tsouna rappelle qu'elle a participé à la traduction de L'Économie pour Les Épicuriens coédité par Daniel Delattre et Jackie Pigeaud (Paris, Gallimard, 2010, Bibliothèque de la Pléiade), il n'est guère fait mention des nouvelles lectures et conjectures dont cette publication a été l'occasion et qui sont disponibles en ligne sur le site du CISPE (http://www.herculanensiacispe.org/langue-francaise.html), à l'exception des pages 72 et 95 (une mention en note 42 , mais pas en note 44).

Ce livre de Philodème appartient à un ensemble plus vaste consacré aux vices et aux vertus opposées et composé d'au moins dix rouleaux-livres. Le titre donné à la fin du PHerc. 1424 est le plus complet de ceux qui sont conservés : Sur les vices et des vertus qui leur sont opposées, des personnes chez qui ils sont présents et des situations dans lesquelles on les rencontre, livre IX (ce qu'on pourrait aussi traduire: Des vices et des vertus qui leur sont opposées, des hommes chez qui ils se rencontrent et des domaines qu'ils concernent; toutefois, aucun sous-titre mentionnant explicitement le domaine de l'économie n'y figure, et le titre qui désigne aujourd'hui couramment l'ouvrage, Peri oikonomias, est simplement déduit du contenu. De fait, le terme grec oikovouia (oikonomia), traduit en anglais américain par property management (《gestion d'une propriété »), y est récurrent, et fait, à juste titre, l'objet d'une explication dès le début de l'introduction (p.xi-xii).

L'introduction, qui reprend dans les grandes lignes le contenu développé par Philodème, sans toujours suivre de façon linéaire la progression du livre, développe cinq points : les thèses de Xénophon et de Théophraste, leurs points communs; la critique qu'en fait Philodème; la position épicurienne en matière d'acquisition et de conservation des biens; la justification de la gestion d'une propriété par une personne qui prétend à une vie conforme à la doctrine épicurienne; le philosophe épicurien comme administrateur ; les sources de revenu. 